\title{
Telerehabilitation in response to constrained physical distance: an opportunity to rethink neurorehabilitative routines
}

\author{
Arturo Nuara ${ }^{1,2,3}$ (1) Maddalena Fabbri-Destro ${ }^{1} \cdot$ Emilia Scalona $^{1} \cdot$ Stefano Elio Lenzi ${ }^{1} \cdot$ Giacomo Rizzolatti $^{1,3}$. \\ Pietro Avanzini ${ }^{1,4}$
}

Received: 9 September 2020 / Revised: 2 January 2021 / Accepted: 4 January 2021 / Published online: 15 January 2021

(c) Springer-Verlag GmbH, DE part of Springer Nature 2021

\begin{abstract}
Ensuring proper dosage of treatment and repetition over time is a major challenge in neurorehabilitation. However, a requirement of physical distancing to date compromises their achievement. While mostly associated to COVID-19, physical distancing is not only required in a pandemic scenario, but also advised for several clinical conditions (e.g. immunocompromised individuals) or forced for specific social contexts (e.g. people living in remote areas worldwide). All these contexts advocate for the implementation of alternative healthcare models. The objective of this perspective is to highlight the benefits of remote administration of rehabilitative treatment, namely telerehabilitation, in counteracting physical distancing barriers in neurorehabilitation. Sustaining boosters of treatment outcome, such as compliance, sustainability, as well as motivation, telerehabilitation may adapt to multiple neurological conditions, with the further advantage of a high potential for individualization to patient's or pathology's specificities. The effectiveness of telerehabilitation can be potentiated by several technologies available to date: virtual reality can recreate realistic environments in which patients may bodily operate, wearable sensors allow to quantitatively monitor the patient's performance, and signal processing may contribute to the prediction of long-term dynamics of patient recovery. Telerehabilitation might spark its advantages far beyond the mere limitation of physical distancing effects, mitigating criticalities of daily neurorehabilitative practice, and thus paving the way to the envision of mixed models of care, where hospital-based procedures are complementarily integrated with telerehabilitative ones.
\end{abstract}

Keywords Physical distancing $\cdot$ Social distancing $\cdot$ Remote rehabilitation $\cdot$ Action observation treatment $\cdot$ Virtual reality

Supplementary Information The online version contains supplementary material available at https://doi.org/10.1007/s0041 5-021-10397-w.

Arturo Nuara

arturo.nuara@gmail.com

1 Consiglio Nazionale delle Ricerche, Istituto di Neuroscienze, via Volturno 39/E, 43125 Parma, Italy

2 Department of Biomedical, Metabolic, and Neuronal Sciences, University of Modena and Reggio Emilia, Modena, Italy

3 Department of Medicine and Surgery, University of Parma, Parma, Italy

4 Istituto Clinico Humanitas, Rozzano, Italy

\section{Introduction}

The amount of training and its reiteration over time are key factors driving a favorable outcome of neurorehabilitative treatments [1,2]. However, keeping a proper dosage and the repetition sustained in the course of time is demanding for all actors of neurorehabilitation. On the one side, healthcare providers must face overscheduling despite a limited availability of equipped spaces and specialized professionals; on the other side, families have to re-organize their daily routines planning travels to rehab facilities, and thus covering high costs in terms of money and time of caregivers [3, 4]. A key challenge in neurorehabilitation practice is to ensure a timely access to cure and its continuity, removing all hindering factors. Among them, constrained physical distancing is one of the most detrimental, since it may affect most of the neurorehabilitative procedures, spanning from the clinician/ patient contact to the joint attendance of treatment spaces. 
In this perspective, we will examine how the constrained physical distancing affects the prosecution of traditional neurorehabilitation programs, hurdling the continuity of rehabilitative pathways. We will propose that telerehabilitation could represent a valuable solution to sustain neurorehabilitative continuity of cure by overcoming social isolation barriers. Previous findings indicated that telerehabilitation may have a positive impact on a range of primary and secondary neurological outcomes $[1,5,6]$ despite the large heterogeneity of interventional parameters and protocol design [5]. Here, our scope is not to ascertain the efficacy of telerehabilitation, but rather to highlight its main advantages and to propose a series of suggestions aimed at maximizing its efficacy and sustainability, in light of modern technology.

In summary, constrained social distance as the one experienced during 2020 pandemic could be seen as an opportunity to rethink current neurorehabilitative routines, envisioning mixed procedures in which face-to-face sessions are integrated and combined with telerehabilitation.

\section{The impact of physical distancing constraints on neurorehabilitation}

Neurorehabilitation is endowed with a peculiar social vocation. Indeed, its activities are grounded on the interaction across patients, caregivers and a multidisciplinary rehabilitative team, and usually take place in spaces hosting multiple patients who can potentially interact with each other. This is why physical distancing constraints severely affect common neurorehabilitative procedures. Exemplars are the consequences of the physical distancing measures combined with changes in healthcare services regulation following the recent COVID-19 pandemic outbreak, as also indicated by several national guidelines[7]. First, most of in-patients treatments have been confined to patient's room, which is intrinsically not conceived for hosting rehabilitation treatment; second, the clinical activities requiring an internal flow (e.g. movement between floors or to reach gym) have been suspended, as well as all the out-patients treatments or those delivered at home by therapists; third, meeting activities and clinical interviews with patient's familiars are currently conducted only by phone or email. As a consequence of such radical measures, rehabilitation programs have been reduced, pursuing only short-term and primary goals, and the activities of the rehabilitative team have been limited to those strictly necessary. Noteworthy, beyond physical distancing measures, also changes in healthcare services access regulation are negatively affecting the access to rehabilitative services during the current pandemic.

Beyond pandemic condition, physical distancing constraints are daily experienced by immunocompromised individuals undergoing neurorehabilitation, e.g. people with aggressive forms of multiple sclerosis undergoing hematopoietic stem cell transplantation [8,9], or frail neurological patients suffering from multimorbidity. Constrained distancing limits rehabilitative options also for patients with infectious disease requiring contact isolation [10]; also in these cases, the access to common spaces (e.g. gym, swimming pools) is restricted, and rehabilitative procedures are bounded to patient's room.

Outside the infectious prevention, physical distancing is a condition experienced also by people living in war zones, incarcerated [11], refugees [12], and, most widely, persons that live in remote areas of the world [13], especially in developing countries [14]. In such cases, the negative impact of distancing and isolation may be exacerbated by the difficulties in transports intrinsic to neurological disability [14].

The interplay between neurological disability and social isolation is worth a discussion. Indeed, disability by itself is an independent factor promoting social isolation for patients $[15,16]$ and caregivers [17]. Thus, all the physical distancing factors discussed above may favor the establishment of a vicious circle in which a poor continuity of cure, limiting the rehabilitative outcome, ends up in feeding physical distancing itself (see Fig. 1).

In this realm, adapting to physical distancing scenarios represents an imperative challenge for neurorehabilitation, whose settings and procedures need to be re-organized to guarantee the achievement of treatment objectives even in the absence of physical closeness among rehabilitation actors. Such solutions may spark the advantages far beyond the mere mitigation of pandemic effects, removing barriers that affect daily neurorehabilitative practice, and thus promoting its sustainability.

\section{Telerehabilitation: an extra-weapon for physical distancing consequences}

The recent advances in Information and Communication Technologies (ICTs) enabled a growing amount of people worldwide to remotely interact at affordable costs, embracing the possibility to join in complex human activities like neurorehabilitation. Telerehabilitation extends treatment delivery beyond the boundaries of traditional healthcare facilities, reaching patients regardless of the distance. This way, telerehabilitation would represent an extra weapon to contrast the detrimental impact of physical distancing on cure accessibility, offering advantages related to both quantitative and qualitative determinants of treatment outcome.

Among the former, continuity of cure is one of the most challenging. Its relevance has been acknowledged in manifold chronic neurological conditions [18], in which patient needs, treatment goals, and procedures have to be periodically reconsidered. Stroke one of the leading causes of 
Fig. 1 The vicious circle involving social distance and neurological disability. Factors hurdling the continuity of rehabilitative pathways impede a favorable outcome, ending up in feeding social distancing itself

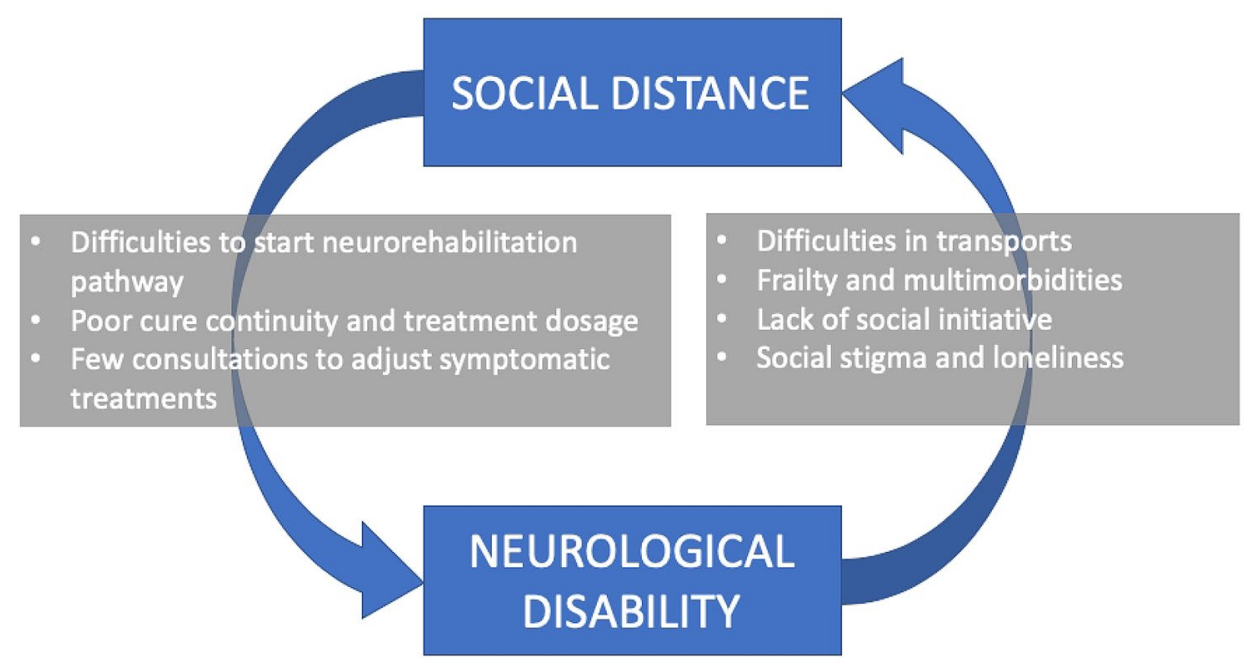

acquired adult disability $[18,19]$ represents a remarkable example of the necessity to implement long-term rehabilitative support. While in stroke patients neurorehabilitation is mostly confined to post-acute phase, the functional improvement is typically distributed over a longer time window. During the months following the post-acute phase, stroke survivors may further take advantage from task-oriented practice promoting adaptive learning and compensation strategies, as well as from specific rehabilitation interventions aimed to improve independence in activities of daily living, social interaction and environmental adaptations [18]. Contrasting the downsizing of rehabilitation providers and the difficulties associated with physical distancing, telerehabilitation represents a suitable solution to sustain the continuity of neurorehabilitation during the post-discharge phase of stroke patients [20], ensuring an excellent compliance to treatment $[1,21,22]$.

Even more so, the same approach may offer advantages in chronic-progressive models of neurological diseases, where rehabilitation has to be periodically delivered lifetime, and adjusted according to the progression of symptoms. Indeed, telerehabilitation has proven feasible and effective in neurological conditions, such as multiple sclerosis (MS) [23], Parkinson's disease[24] and Alzheimer's disease[25]. The capacity of telerehabilitation to promote continuity of cure extends to disadvantaged social contexts, and have been remarked in explorative studies conducted in resource-limited rural settings of developing countries, where neurorehabilitation is challenged by lack of trained personnel and huge geographic barriers [14].

Another quantitative factor easily affordable by telerehabilitation is the maximization of treatment dosage. A large body of studies evidenced that greater amounts of practice amplify neural plasticity processes, reflecting in a faster and higher improvement of behavioral functions $[2,26,27]$. Such a dose-dependent effect of training also applies to neurorehabilitation, with the time scheduled for therapy positively correlated with the therapy outcome [2]. Telerehabilitation may help to overcome several criticalities for treatment dosage even beyond those strictly due to physical distancing. Indeed, while limited resources and personnel make the rationing of traditional cure unavoidable, telerehabilitation would sustain the treatment dosage, counteracting the limited amount of exercises usually delivered in hospitalized settings [2, 28].

Another feature of telerehabilitation potentially boosting effectiveness is its continuous availability, which in turn sets no obstacles to the treatment delivery during the sensitive window for each patient. On the contrary, in common hospitalized settings, the long waiting lists often impede the administration of treatment in proper time, making it available only belatedly, i.e. not well-matching the patient's window of improvement.

A key qualitative factor for a positive outcome is patient's motivation [29-31], both in its external and internal components [29]. The former-related to the engaging properties of the administered tasks - can be handled by means of gamification elements and virtual reality, making the rehabilitative experience attractive $[20,22,32$, 33]. The latter-stemming from patient's own awareness of improvements-may be obtained through the dynamic adjustment of rehabilitation goal [34], as well as by means of the delivery of performance-dependent and rewarding feedbacks [22].

Another point of strength of telerehabilitation relies on its home-based setting. Indeed, besides the logistic facilitations for patients and caregivers, the possibility to act in a familiar scenario may boost participation to procedures, especially in categories of subjects like children suffering from cerebral palsy $[22,30,35]$ and cognitively impaired elder people [25], whose behavior and commitment to tasks may deteriorate when acting in unfamiliar environments [36]. 
Noteworthy, to be applied in a wide population and over prolonged times, treatments have to display an adequate sustainability. Telerehabilitation fulfills this requirement, thanks to its capacity to relieve the care burden affecting caregivers and healthcare providers, impacting on the global economic cost by reducing travel expenses and decreasing loss of productivity time [37], in particular when applied to larger rural areas $[38,39]$.

Besides cost-effectiveness gains [38-40], advanced telerehabilitation protocols have shown satisfactory profiles of feasibility. This latter has been extensively investigated in a recent study on children suffering from unilateral cerebral palsy, where outcomes, such as customization of exercises, acceptability at home, required effort and technological suitability, highlighted a feasible profile of the intervention [30].

All these factors may deeply contribute to establishing coherence between patient's behavior and prescribed indications, boosting a major factor of neurorehabilitation outcome, i.e. compliance to treatment. In this regard, a critical issue may be represented by the verification of patient's adherence to expected procedures. Even such a monitoring activity may be conducted via ICTs, using dedicated online daily questionnaires and diaries [22], as well as session video recordings acquired by means of webcams installed at patient's home [22, 30].

\section{Limitations}

Aside to the above-mentioned strenghts, telerehabilitation presents some weaknesses (see Peretti et al. [41]). The first limitation is the absence of in-person session monitoring, potentially impacting on the verification of safety of procedures. Any solution allowing for an an online and remote monitoring of the patient's performance (webcams or sensors), the involvement and education of caregivers, and when possible the periodical alternation with in-person sessions, would temperate this criticality. The second issue concerns the heterogeneous evidence in terms of procedures, outcomes and effectiveness, narrowing a broader applicability. A recent metanalysis conducted by Laver and coworkers [42] showed that, despite the increasing number of studies testing the efficacy of telerehabilitation in patients with stroke, it is hard to reach conclusions on its effectiveness due to the considerable variability across studies in terms of interventions and outcome measures. However, since several studies comparing telerehabilitation and in-person treatment did not found outcomes significantly different between groups, the authors suggest that at least in stroke, telerehabilitation efficacy would be not inferior to that of traditional rehabilitation, with estimated effect sizes in favour of telerehabilitation ranging from $0.48[-1.36,2.32]$ for balance to 1.23 [-2.17,4.64] for upper-limb motor function [42]. Future research would benefit from the adoption of standardized procedures and outcome measures, which would in turn enable the comparison across studies and the delivery of more robust recommendations for telerehabilitative practice. For this purpose, the routinary adoption of telerehabilitative procedures during current pandemic would represent an unique opportunity to collect robust data on telerehabilitation, to conduct a rigorous investigation of its cost-effectiveness, and to reach solid results on its feasibility and efficacy.

Since advanced telerehabilitation is associated with an enhanced use of technologies as VR and sensors, it is worth to mention also the benefits and disadvntages specifically linked to their adoption. When dealing with technologically advanced procedures, a further limitation regards the low acceptance by people with poor confidence with digital technology. This issue, potentially involving both patients and providers, may be counteracted by a preliminary training and by implementing easily affordable procedures. Of note, scarce confidence with digital technology would likely reduce in the next decades, when digital natives will progressively represent an even larger slice of the overall population.

The main advantages and weaknesses of advanced telerehabilitation, as well as the measures aimed at counteracting limitations, are summarized in Table 1.

\section{Suggestions for telerehabilitation implementation}

As a general principle, the ideal telerehabilitative treatment should be intensive, warrant a proper repetition over time and sustain patient motivation and interest $[1,6]$. Indeed, these are all factors contributing to maximize the neurorehabilitative treatment outcome.

The establishment of telerehabilitation in the daily routines of neurological patients necessarily implies the definition of key aspects impacting on its efficacy and sustainability. These include (a) the identification of patients eligible for receiving treatment, in turn informing the individualization of procedures according to patient's or pathology's specificities (b) the choice of telerehabilitative approach, c) the definition of the setting, including devices and sensors collecting data relative to patient performance, and finally, (d) the selection of signal processing procedures suitable to provide insights on the current patient performance, and possibly predict future developments. These points will be addressed separately in the next sections.

\section{Patient eligibility and treatment individualization}

With the exception of few procedures where physical contact between patients and therapists is essential, such as manipulative treatments, most of common neurological impairments 
Table 1 Main advantages and limitations of technologically-advanced telerehabilitation

\begin{tabular}{|c|c|c|c|}
\hline & Advantages & Limitations & Countermeasures \\
\hline \multirow[t]{3}{*}{ Telerehabilitation } & \multirow{3}{*}{$\begin{array}{l}\text { Acting in a com- } \\
\text { fortable, home- } \\
\text { based, setting } \\
\text { Possibility to reach } \\
\text { remote locations } \\
\text { Dynamic adapt- } \\
\text { ability to patient's } \\
\text { profile and } \\
\text { environment } \\
\text { Reduced transports } \\
\text { Increased sustain- } \\
\text { ability of reha- } \\
\text { bilitative pathway }\end{array}$} & Absence of in-person monitoring & $\begin{array}{l}\text { Adoption of technologically-advanced } \\
\text { remote monitoring } \\
\text { Caregiver's active involvement and educa- } \\
\text { tion }\end{array}$ \\
\hline & & $\begin{array}{l}\text { Absence of physical, face-to-face contact } \\
\text { with healthcare personnel } \\
\text { Difficult symptomatic therapy adjust- } \\
\text { ment } \\
\text { Difficult evaluation and management of } \\
\text { spasticiy }\end{array}$ & $\begin{array}{l}\text { Adoption of a "mixed model", alternating } \\
\text { traditional face-to-face neurorehabilita- } \\
\text { tion with telerehabilitation }\end{array}$ \\
\hline & & $\begin{array}{l}\text { Heterogeneous evidences on telereha- } \\
\text { bilitative procedures, settings and } \\
\text { outcomes }\end{array}$ & $\begin{array}{l}\text { Adoption of standardized procedures and } \\
\text { outcome measures } \\
\text { Systematic, research-oriented collection of } \\
\text { data during telerehabilitation protocols }\end{array}$ \\
\hline $\begin{array}{l}\text { Use of technologically-advanced } \\
\text { devices (e.g. Virtual Reality, } \\
\text { sensors) }\end{array}$ & $\begin{array}{l}\text { Gamificated } \\
\text { features boosting } \\
\text { motivation and } \\
\text { engagement } \\
\text { Creation of immer- } \\
\text { sive or aug- } \\
\text { mented virtual } \\
\text { scenarios } \\
\text { Online feedback } \\
\text { delivery } \\
\text { Acquisition of } \\
\text { biomarkers via } \\
\text { dedicated sensors } \\
\text { Administration in } \\
\text { ecological, real- } \\
\text { life environment } \\
\text { Patient's digital } \\
\text { phenotyping }\end{array}$ & $\begin{array}{l}\text { Low acceptance by people with poor } \\
\text { confidence with technology } \\
\text { Need of prompt technical assistance in } \\
\text { case of malfunctioning } \\
\text { Side effects related to virtual reality } \\
\text { (motion sickness syndrome) } \\
\text { Increase of equipment costs }\end{array}$ & $\begin{array}{l}\text { Preliminary patient's training } \\
\text { Implementation of easy-to-use, cost- } \\
\text { affordable and safe equipment }\end{array}$ \\
\hline
\end{tabular}

may take advantage of telerehabilitation, whose procedures need to be tuned to patient's own impairments and rehabilitation objectives.

In the realm of stroke-related disability, upper-extremities motor training represents one of the most suitable scopes for telerehabilitation[1, 6]. Here, goal-oriented approaches have proven effective in inducing motor improvements similar to those produced by traditional in-clinic rehabilitation. Besides upper-limbs motor domain, also balance and gait symptoms due to stroke may benefit from telerehabilitative treatment [43]. However, the implementation of domicilary setting for walking training is more challenging due to the limited space and to safety concerns. In this regard, the use of computer screens or projectors as viewing devices in place of immersive visors, and more generally the selection of wireless devices could prevent the risk of falls during training procedures [44].

The design of stroke telerehabilitative procedures should take into account the presence of associated symptoms potentially affecting patient's adherence to treatment. Among them, spasticity - affecting about one-third of patients with stroke[45] might support the choice of customized and easy-to-handle haptic devices, as well as the adoption of sensors compatible with spastic hypertonia (e.g. sensorized gloves should be avoided). Moving to the visual domain, when symptoms like hemianopsia or unilateral spatial neglect concur, the design of stimuli and their administration need to be adapted to patient's own visual skills [46].

Among neurodegenerative movement disorders, Parkinson's disease (PD) is the one in which telerehabilitation is most adopted. In patients with PD, the remote delivery of motor tasks associated to visual, auditory or haptic feedbacks may be effective to improve postural stability and walking skills [47, 48]. Here, safety recommendations similar to those indicated above for gait training in stroke patients should be embraced. Interestingly, the potential application of telerehabilitation in PD goes beyond the training of gait-related abilities, encompassing the possibility to target upper-limb motricity [24] or phonological skills [49]. Noteworthy, people with PD undergoing Levodopa pharmacological treatment should be instructed to perform training only during the ON state to limit the negative impact of motor fluctuation on telerehabilitative sessions [50]. 
Moving to younger subjects, telerehabilitation is facilitated by the higher degree of confidence with technology. This would be the case of people with MS. Consistently with the heterogeneous clinical manifestations of MS, here telerehabilitation can target multiple motor fields, such as balance [51], gait [52], fatiguability [53], as well as cognitive skills like executive functions [54], verbal fluency and memory [55] (see also Di Tella et al. for a systematic review [56]).

Finally, in the realm of pediatric population, the remote administration of rehabilitative treatments may take advantage from the possibility to enrich sessions with gamificated features. This aspect jointly with the instrinsic benefit of acting in a home-based setting might be exploited in children with cerebral palsy, administering motivating task-oriented exercises able to improve upper limbs motor function [22, $30,57]$.

Despite its encouraging potentialities, the administration of telerehabilitation may present relevant challenges in patients suffering from cognitive disturbances [25], where understanding of procedures may be impaired, and then adherence to treatment suboptimal.

Another instance where the adoption of telerehabilitation should be cautiously considered concerns patients with poor confidence or aversion toward technology. Such conditions more frequent in elderly people may be mitigated by a proper preliminary training, and by the choice of an easy-to-use setup.

Finally, patient eligibility has to deal with neurological symptoms severity or possible comorbidities. For instance, verbal impairments impeding a proper communication with cure providers, as well as the occurrence of relevant cardiovascular comorbidities requiring continuous and supervised monitoring during active exercises could contraindicate the participation in telerehabilitation.

\section{Telerehabilitative approaches}

\section{Task-oriented motor rehabilitation}

Most of evidence on neurological telerehabilitation effectiveness come from randomized-controlled studies where task-oriented approaches have been remotely applied in stroke patients with upper-limb motor impairment [6, 20, 42]. It is worth to note that task-oriented methods may be also fruitfully applied to other brain injuries [58] and neurodegenerative conditions [59].

Task-oriented approach consists in administering goaldirected tasks targeting various motor control features, spanning from movement speed to range of motion (see Cramer et al. [18] for an exemplar repertoire of exercises). Despite traditionally targeting upper limbs $[6,20]$, goal-oriented remote approaches have been recently adopted in the balance and gait rehabilitation field, where the introduction of low-cost infra-red camera and portable balance boards allow to deliver home-based training to people with MS [51], Parkinson's disease [60] and stroke [43].

As a general rule of thumb, task-oriented exercises should meet the following criteria: (1) being challenging and meaningful, (2) addressing relevant and multiple impairments, (3) enhancing specific motor abilities through overload, (4) being endowed with goal-directedness in movement organization. Here, the major driver of task-specific self-confidence is represented by the successful performance accomplishment (see Winstein et al. [61]). Associated gamification features and feedbacks delivery may emphasize this latter factor, ultimately boosting task-oriented effectiveness [20].

\section{Action Observation Treatment}

Despite being groupable among task-oriented approaches, Action Observation Treatment (AOT) deserves a special consideration due to its methodological peculiarities, making it a paradigmatic framework for telerehabilitation. AOT is based on the notion that the observation of an action, followed by its imagination and executional attempt, is able to promote neuroplasticity processes underlying motor functional improvement [62]. Its efficacy has been demonstrated in a wide range of neurological disorders, such as stroke [63], Parkinson's disease [64], multiple sclerosis [65] and cerebral palsy [30].

Being grounded on the delivery of visual stimuli depicting actions to be trained, AOT is endowed with a special vocation for remote administration. Indeed, AOT has been recently opened to telerehabilitation, especially in pediatric population [22, 30, 57]. Nuara et al. [22] applied a homebased, peer-to-peer AOT to 20 children with cerebral palsy (CP) with upper-limb impairment. During AOT remote sessions, participants had to observe and then imitate a wizard performing dexterity-demanding magic tricks. Kinematics were monitored via a markerless infrared system, and reinforcing feedbacks were provided upon the use of the impaired hand. Subsequently, a peer-to-peer live videosession to practice the same exercises took place. Following treatment, an improvement in hand motor abilities was found. Of note, peer-to-peer difference in hand motor ability was correlated to the amount of improvement, indicating that it is preferable for a child to observe a leading peer with higher motor skills. Beyond proving the feasibility of telerehabilitative approach for AOT, this study showed that in a "dual rehabilitation model", patients could simultaneously act as "beneficiaries" and "providers" within the motor rehabilitation process.

Scaling up the remote and peer-to-peer approach for dual rehabilitation, one could envision the realization of a wide network of patients undergoing interactive and remote AOT. Here, each user could in turn improve his skills by 
interacting with a more capable peer, or act as a trainer towards a more impaired individual. Beyond promoting a greater motor resonance [22, 66, 67], the interplay across patients experiencing the same symptoms would favour beneficial social instances, like reciprocal encouragement and other friendly exchanges. Besides the high sustainability, the peer-to-peer approach could be easily extended to other neurological conditions in which AOT has proven effective, opening technologically advanced telerehabilitation to novel, social-enriched scenarios.

\section{Cognitive telerehabilitation}

Cognitive rehabilitation includes a wide group of interventions aimed at consolidating or recovering previously acquired patterns of behavior, or at establishing new cognitive abilities compensating for impaired neurological systems [68]. Here, adopting telerehabilitation would ease the achievement of key factors of cognitive treatment efficacy, such as training intensivity, prolongation over time and engagement, ultimately empowering patient's ability to transfer cognitive skills from virtual to real world [69]. Studies demonstrating the feasibility and effectiveness of cognitive rehabilitation have been mostly conducted on people suffering from neurodegenerative diseases (see Cotelli et al. [25] for a recent systematic review). However, there are also evidences that remote administration of cognitive rehabilitation can ameliorate the cognitive outcome of post-surgical patients with brain tumors [70], as well as of people with stroke [71] and multiple sclerosis [72].

Recent approaches in cognitive telerehabilitation encompass the use of interactive applications pursuing learning and education as primary objectives (i.e. serious games). Combining a challenging and rewarding nature with affordable costs, serious games can be intensively administered in the familiar environment of patient's own home. Their adoption showed promising results in improving cognitive symptoms in Alzheimer disease [73], stroke [74] and cerebral palsy [75]. However, their diffusion is restricted because of the poor customization and the limited theorethical ground driving their development. For this reason, a growing effort is ongoing to tailor serious games to the clinical features of people with specific cognitive impairments [69].

\section{Patient and caregiver's education}

Besides the administration of active treatments, neurological telerehabilitation should incorporate complementary health services for the achievement of disease-specific priorities able to affect long-term outcomes [6, 20, 76]. Among them, the education of patients about relevant aspects of their own disease may be crucial for health-status awareness, for secondary prevention $[6,20]$, and for the adoption of proper life-styles aimed at improving quality of life [77]. This possibility has been recently exploited for patients with stroke, where daily "education pills" have been embedded in the telerehabilitative path to optimize functional status, boost motivation and prevent secondary cerebrovascular events [20]. Such an educational approach could be extended also to neurodegenerative conditions, where life-style and dietary measures may potentially impact on disease progression and quality of life $[78,79]$.

\section{Telerehabilitation settings}

The ideal rehabilitation setting should combine the highest portability and smartness on the patient side, with the highest efficiency on the healthcare provider side. For simplicity, from here on, we will refer to these sides as patient and therapist, respectively.

The main elements comprising the patient-setting are: a computer connected to internet, devices for audio-visual presentation, and wearable sensors interfacing with the computer for tracking the patient's performance.

Concerning hardware, current solutions maximize the portability and lightness of a tele-rehabilitative setup. Indeed, workstations with a Local Area Network (LAN) connection can be easily replaced to date by portable devices (laptops, tablets, or even smartphones) continuously connected to wireless networks. Although well-fitting with the notion of home-based telerehabilitation, such solutions would allow a full-portability of the telerehabilitation setup, making patients capable to adhere to treatment at any time and any place.

While the above-mentioned devices allow the presentation of multimedia content, several rehabilitative practices adopted virtual reality (VR) to recreate realistic and threedimensional environments in which patients may bodily operate. Among VR techniques, fully immersive VR is based on a completely computer-generated environment built to evoke the perception to be physically present in a virtual world. Here, the viewing medium is generally represented by a head-mounted visor. Conversely, in mixed reality (MR), real and virtual objects coexist, interacting in a mixed environment. Closer to the real-world scenario, augmented reality (AR) enhances the sensory experience of the real environment introducing computer-generated elements, encompassing the adoption of common devices like smartphones or tablets as viewing media (see Table 2 for a summary of features and administration modalities of main VR technologies). Noteworthy, the different degrees of immersion and physical interaction [80, 81], as well as viewing devices, need to be chosen according to patient's specific features and rehabilitative aims. For example, in cognitive rehabilitation, the VR system has to be designed to ensure a comfortable and familiar virtual experience [82]. Mixed 
Table 2 Basic features and administration modalities of the principal virtual reality technologies

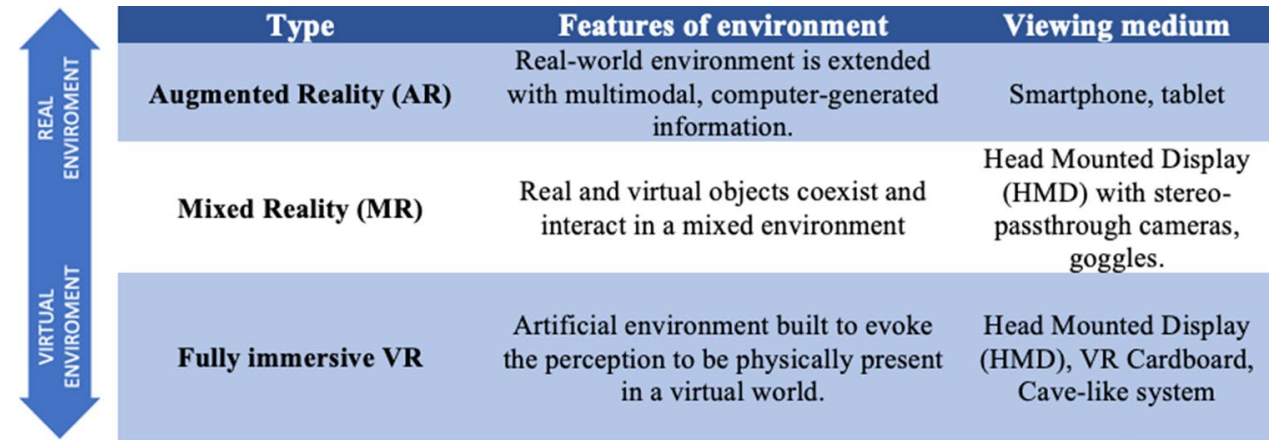

reality would best fit in this case, since patient perception to attend procedures at his/her own home might boost the compliance to sessions, avoiding the dispersion of attentive resources, reducing anxiety and ultimately favoring treatment outcome. Especially, but not exclusively, in cognitively impaired patients, preliminary-assisted training aimed at verifying the comprehension of the task and the tolerability to the procedure, is advised thus ensuring users' comfort with the VR technology.

VR technologies have been fruitfully applied to several neurological conditions, including stroke [83-86], Parkinson's disease [50, 87], multiple sclerosis [51, 88] and cerebral palsy $[89,90]$, showing an overall satisfactory profile of feasibility and efficacy [91]. Modern VR-based systems (e.g. headsets integrated with haptic, auditory and visual feedbacks) allow the administration of a multi-modal, fully immersive stimulation, giving to the patient the vivid perception to be physically present in the virtual environment. Sometimes, VR users may experience symptoms of motion sickness (commonly referred as "VR sickness" or "cybersickness"), including dizziness, fatigue, disorientation and nausea. Several preventive measures have been proposed to mitigate such side effects, like providing multimodal stimulation, using dynamic adjustment of depth of field, increase the fidelity of virtual scenarios with sinchronous and multimodal stimuli (see Chang et al. [92] for a review). Novel allin-one VR solutions merging the computer and the viewing device within a wireless headset, may include optical tracking systems and advanced visual adjustments to prevent the insurgence of dizziness and motion sickness, thus making VR technology applicable for a larger number of patients.

In a recent metanalysis, Howard [91] identified three major factors linked to the efficacy of VR-based neurorehabilitation. The first one-namely "increased excitement"regards the patient attitude to be engaged during the treatment procedures. Boosting attention and motivation, such a positive reaction may be favored by the adoption of novel immersive devices, the delivery of rewarding feedbacks and specific "gamification" features [93, 94]. The second one is the "increased physical fidelity", i.e. the ability to transpose into the "real-life" tasks performed during the session. In this regard, mixed-reality-based systems could sustain a realistic perception of the environment by fostering a natural performance on behalf of patients. The third factor is the "increased cognitive fidelity", that is the capacity of VRbased treatments to ground psychological processes in scenarios whose complexity resembles everyday life [95, 96].

Beyond informing the patient, the ideal telerehabilitation setup should inform about the patient. Direct monitoring of patient performance is fundamental to verify the adherence to treatment, to ensure safety, and to monitor recovery trajectory. While the first two aspects can be fully achieved by means of teleconference systems, the recovery trajectory needs to be objectively quantified estimating subtle changes in patient's performance by integrating specific monitoring devices in the rehabilitation system.

Portable mechatronic devices (i.e. haptic joysticks) represent one of the earlier and cost-effective solutions for upperlimb telerehabilitation. They can apply forces and measure hand position, thus indirectly quantifying the user performance [97]. However, these devices allow only few types of movement, hindering the possibility to evaluate fine motor actions like grasping or finger apposition [98]. To overcome these limitations, systems based on sensorized gloves have been developed and tested in neurological patients [89, 99]. Despite the promising results of their adoption in hand motor training protocols $[89,99]$, the potential dependence on caregivers for the wearing may represent a barrier for their use. Moreover, the exclusive monitoring of the hand does not permit the evaluation of concomitant postural attitudes of more proximal segments of the upper limb, as well as of the trunk [100]. In turn, Inertial Measurement Units (IMUs) systems are able to reconstruct accurately complex and multi-segmentary body postures also in outdoor environments. Nevertheless, these systems are often difficult to wear by neurological patients without assistance. The maximal independence of the patient could be ensured by optical marker-less solutions (e.g. portable infrared camera sensors), which however are mainly suited to track large movements [101] 
Concerning trunk and lower limbs kinematic evaluation, beyond IMUs, simpler wearable step counters (i.e. podometers) may provide information about gait parameters like walking speed, cadence, double support and stride with a reliability comparable to the gold standard for gait analysis $[102,103]$. In addition, even smartphones can acquire useful objective data about gait and balance, being equipped with accelerometers and gyroscopes to detect body parameters, such as falls, postural sway, gait performance, and balance stability [104, 105].

In summary, the choice of the appropriate sensor should be based on patient's neurological impairment, the primary outcome, the possibilities of assistance from the caregivers, and costs affordability. Moreover, to favor the detailing of patient's clinical picture, multiple and complementary types of sensors could be simultaneously adopted.

Once the patient-setting has been designed, an equally relevant part to take care of is the therapist-setting, i.e. the architecture enabling the home-based setup to download information relevant to the treatment delivery, and to upload data concerning the patient performance. Ideally, an online platform should be accessible $24 / 7$ by both patients and clinicians, with secure accounts adhering to privacy international rules. The former can start a new session at their convenience, having stimuli and procedure automatically downloaded according to clinical prescription and to their own rehabilitative history. The latter, in turn, can access the system either to monitor online the patient performance (e.g. during the first home-based session or after relevant treatment change), or to review offline the same performance in light of the whole medical history.

Beyond facing security issues, the bidirectional data transfer between the patient- and therapist-settings should keep into account the possibility of connectional malfunctions, thus accounting for proper buffering and/or offline data storage strategies.

\section{Data processing}

The use of sensors presents three main cascade advantages. The first is the online monitoring of patient's performance allowing clinicians and therapists to provide within-session feedbacks to patients or caregivers. Even if a simple webcam would be enough to detect large anomalies in behavioral performance of the patients, sensors could instantly signal subtle anomalies relative to previous history and/or to normative data.

A second advantage is the possibility to deliver online feedbacks aimed at encouraging appropriate patient's behavior[22], or conversely at discouraging unsuitable ones; in this regard, the adoption of strategies integrating sensors and virtual reality (e.g. the online visual amplification of patient's errors) may boost the learning process [80], biasing patient's behavior toward the correct one.

Last but not least, the therapist-platform can be embedded with signal processing tools capable to identify relevant features about individual patient performance and history, and to compare them against data from other patients matched for clinical conditions. In addition, to provide relevant insights on the long-term dynamics of patient's performance, this approach would pave the way to digital phenotyping [106], and the subsequent implementation of machine-learning models aimed to predict functional outcomes. Such information would ultimately support choices and adjustments by the clinicians, thus maximizing the beneficial effects of the whole rehabilitative pathway.

\section{Conclusion}

Thanks to modern technology, an exclusive telerehabilitation represents the only valuable and scalable solution in case of constrained and persistent social distance requirements, making patients act in virtual scenarios while remotely interacting with clinicians.

The lesson taught by COVID-19 pandemic, however, could partially apply also to daily neurorehabilitation routines, so to relieve the care burden around neurological patients and their management. Indeed, while face-to-face therapeutic interactions represent an irreplaceable element in the relationship between patients and healthcare providers, telerehabilitative sessions could act as the missing pieces of the puzzle leading to an optimal continuity of cure.

In such a "mixed" model of neurorehabilitative care, hospital-based procedures (e.g. post-acute protocols, clinical-neurophysiological evaluations, intensive training, etc.) might be used mainly to forge the scaffold of the rehabilitative program, while telerehabilitation could be prevalent in the long-term consolidation of functional progresses [107]. The development of this model requires the synergistic involvement of clinicians, therapists, engineers, developers along with caregivers and patients to promote the overall sustainability and effectiveness of the rehabilitative pathway.

\section{Compliance with ethical standards}

Conflicts of interest All the Authors do not present conflict of interest. All authors approved the submission of the article. 


\section{References}

1. Cramer SC, Sur M, Dobkin BH et al (2011) Harnessing neuroplasticity for clinical applications. Brain 134:1591-1609

2. Lohse KR, Lang CE, Boyd LA (2014) Is more better? Using metadata to explore dose-response relationships in stroke rehabilitation. Stroke 45:2053-2058

3. Maresova P, Lee S, Fadeyi OO et al (2020) The social and economic burden on family caregivers for older adults in the Czech Republic. BMC Geriatr. https://doi.org/10.1186/s12877-02001571-2

4. Cavallo MC, Fattore G (1997) The economic and social burden of Alzheimer disease on families in the Lombardy region of Italy. Alzheimer Dis Assoc Disord 11:184-190

5. Appleby E, Gill ST, Hayes LK et al (2019) Effectiveness of telerehabilitation in the management of adults with stroke: a systematic review. PLoS ONE 14:e0225150

6. Dodakian L, McKenzie AL, Le V et al (2017) A home-based telerehabilitation program for patients with stroke. Neurorehabil Neural Repair 31:923-933

7. Bartolo M, Intiso D, Lentino C et al (2020) Urgent measures for the containment of the coronavirus (Covid-19) epidemic in the neurorehabilitation/rehabilitation departments in the phase of maximum expansion of the epidemic. Front Neurol. https://doi. org/10.3389/fneur.2020.00423

8. Celius EG (2017) Infections in patients with multiple sclerosis: Implications for disease-modifying therapy. Acta Neurol Scand 136:34-36

9. For the European Society for Blood and Marrow Transplantation (EBMT) Autoimmune Diseases Working Party (ADWP) and the Joint Accreditation Committee of the International Society for Cellular Therapy (ISCT) and EBMT (JACIE), Sharrack B, Saccardi R et al (2020) Autologous haematopoietic stem cell transplantation and other cellular therapy in multiple sclerosis and immune-mediated neurological diseases: updated guidelines and recommendations from the EBMT Autoimmune Diseases Working Party (ADWP) and the Joint Accreditation Committee of EBMT and ISCT (JACIE). Bone Marrow Transpl 55:283-306

10. Vonberg R-P, Kuijper EJ, Wilcox MH et al (2008) Infection control measures to limit the spread of Clostridium difficile. Clin Microbiol Infect 14:2-20

11. Akiyama MJ, Spaulding AC, Rich JD (2020) Flattening the curve for incarcerated populations Covid-19 in jails and prisons. $\mathrm{N}$ Engl J Med. https://doi.org/10.1056/NEJMp2005687

12. Strang AB, Quinn N (2019) Integration or isolation? Refugees' social connections and wellbeing. J Refug Stud. https://doi. org/10.1093/jrs/fez040

13. Repke MA, Ipsen C (2020) Differences in social connectedness and perceived isolation among rural and urban adults with disabilities. Disabil Health J 13:100829

14. Sarfo FS, Adusei N, Ampofo M et al (2018) Pilot trial of a telerehab intervention to improve outcomes after stroke in Ghana: a feasibility and user satisfaction study. J Neurol Sci 387:94-97

15. Makizako H, Shimada H, Tsutsumimoto K et al (2015) Social frailty in community-dwelling older adults as a risk factor for disability. J Am Med Dir Assoc 16(1003):e7-11

16. Burholt V, Windle G, Morgan DJ et al (2017) A Social model of loneliness: the roles of disability, social resources, and cognitive impairment. Gerontologist 57:1020-1030

17. Baumgardner DJ (2019) Social isolation among families caring for children with disabilities. J Patient Cent Res Rev 6:229-232

18. Langhorne P, Bernhardt J, Kwakkel G (2011) Stroke rehabilitation. Lancet Lond Engl 377:1693-1702

19. Bonita R, Mendis S, Truelsen T et al (2004) The global stroke initiative. Lancet Neurol 3:391-393
20. Cramer SC, Dodakian L, Le V et al (2019) Efficacy of homebased telerehabilitation vs. in-clinic therapy for adults after stroke: a randomized clinical trial. JAMA Neurol 76:1079

21. Covert LT, Slevin JT, Hatterman J (2018) The effect of telerehabilitation on missed appointment rates. Int J Telerehabilitation 10:65-72

22. Nuara A, Avanzini P, Rizzolatti G et al (2019) Efficacy of a home-based platform for child-to-child interaction on hand motor function in unilateral cerebral palsy. Dev Med Child Neurol. https://doi.org/10.1111/dmcn.14262

23. Amatya B, Galea MP, Kesselring J et al (2015) Effectiveness of telerehabilitation interventions in persons with multiple sclerosis: a systematic review. Mult Scler Relat Disord 4:358-369

24. Cikajlo I, Hukić A, Dolinšek I et al (2018) Can telerehabilitation games lead to functional improvement of upper extremities in individuals with Parkinson's disease? Int J Rehabil Res 41:230-238

25. Cotelli M, Manenti R, Brambilla M et al (2019) Cognitive telerehabilitation in mild cognitive impairment, Alzheimer's disease and frontotemporal dementia: a systematic review. J Telemed Telecare 25:67-79

26. Doyon J, Song AW, Karni A et al (2002) Experience-dependent changes in cerebellar contributions to motor sequence learning. Proc Natl Acad Sci 99:1017-1022

27. Nudo RJ, Milliken GW, Jenkins WM et al (1996) Use-dependent alterations of movement representations in primary motor cortex of adult squirrel monkeys. J Neurosci Off J Soc Neurosci 16:785-807

28. Lang CE, MacDonald JR, Reisman DS et al (2009) Observation of amounts of movement practice provided during stroke rehabilitation. Arch Phys Med Rehabil 90:1692-1698

29. Chen Y, Abel KT, Janecek JT et al (2019) Home-based technologies for stroke rehabilitation: a systematic review. Int J Med Inf 123:11-22

30. Beani E, Menici V, Ferrari A et al (2020) Feasibility of a homebased action observation training for children with unilateral cerebral palsy: an explorative study. Front Neurol. https://doi. org/10.3389/fneur.2020.00016

31. Barak Ventura R, Nakayama S, Raghavan P et al (2019) The role of social interactions in motor performance: feasibility study toward enhanced motivation in telerehabilitation. J Med Internet Res 21:e12708

32. Donoso Brown EV, Dudgeon BJ, Gutman K et al (2015) Understanding upper extremity home programs and the use of gaming technology for persons after stroke. Disabil Health J 8:507-513

33. Proffitt R, Lange B (2015) The feasibility of a customized, inhome, game-based stroke exercise program using the microsoft kinect sensor. Int J Telerehabilitation 7:23-34

34. Wolf SL, Sahu K, Bay RC et al (2015) The HAAPI (Home Arm Assistance Progression Initiative) trial: a novel robotics delivery approach in stroke rehabilitation. Neurorehabil Neural Repair 29:958-968

35. Kirkpatrick E, Pearse J, James P et al (2016) Effect of parentdelivered action observation therapy on upper limb function in unilateral cerebral palsy: a randomized controlled trial. Dev Med Child Neurol 58:1049-1056

36. Verbeek H, van Rossum E, Zwakhalen SMG et al (2009) Small, homelike care environments for older people with dementia: a literature review. Int Psychogeriatr 21:252

37. Dimmick SL, Mustaleski C, Burgiss SG et al (2000) A case study of benefits and potential savings in rural home telemedicine. Home Healthc Nurse 18:124-135

38. Barker GP, Krupinski EA, Schellenberg B et al (2004) Expense comparison of a telemedicine practice versus a traditional clinical practice. Telemed J E Health 10:376-380 
39. Scheideman-Miller C, Clark PG, Moorad A, et al (2003) Efficacy and sustainability of a telerehabilitation program. In: 36th Annual Hawaii International Conference on System Sciences, 2003. Proceedings of the. Big Island, HI, USA: IEEE, $p 11$

40. Calabrò RS, Bramanti A, Garzon M et al (2018) Telerehabilitation in individuals with severe acquired brain injury: rationale, study design, and methodology. Medicine (Baltimore) 97:e13292

41. Peretti A, Amenta F, Tayebati SK et al (2017) Telerehabilitation: review of the state-of-the-art and areas of application. JMIR Rehabil Assist Technol 4:e7-e7

42. Laver KE, Adey-Wakeling Z, Crotty M et al (2020) Telerehabilitation services for stroke. Cochrane Database Syst Rev. https:// doi.org/10.1002/14651858.CD010255.pub3

43. Lloréns R, Noé E, Colomer C et al (2015) Effectiveness, usability, and cost-benefit of a virtual reality-based telerehabilitation program for balance recovery after stroke: a randomized controlled trial. Arch Phys Med Rehabil 96(418-425):e2

44. Schröder J, van Criekinge T, Embrechts E et al (2019) Combining the benefits of tele-rehabilitation and virtual reality-based balance training: a systematic review on feasibility and effectiveness. Disabil Rehabil Assist Technol 14:2-11

45. Watkins CL, Leathley MJ, Gregson JM et al (2002) Prevalence of spasticity post stroke. Clin Rehabil 16:515-522

46. Pedroli E, Serino S, Cipresso P et al (2015) Assessment and rehabilitation of neglect using virtual reality: a systematic review. Front Behav Neurosci 9:1-15

47. Cano Porras D, Siemonsma P, Inzelberg R et al (2018) Advantages of virtual reality in the rehabilitation of balance and gait: systematic review. Neurology 90:1017-1025

48. Canning CG, Allen NE, Nackaerts E et al (2020) Virtual reality in research and rehabilitation of gait and balance in Parkinson disease. Nat Rev Neurol 16:409-425

49. Quinn R, Park S, Theodoros D et al (2019) Delivering group speech maintenance therapy via telerehabilitation to people with Parkinson's disease: a pilot study. Int J Speech Lang Pathol 21:385-394

50. Gandolfi M, Geroin C, Dimitrova E et al (2017) Virtual reality telerehabilitation for postural instability in parkinson's disease: a multicenter, single-blind, randomized. Control Trial BioMed Res Int. https://doi.org/10.1155/2017/7962826

51. Prosperini L, Fortuna D, Giannì C et al (2013) Home-based balance training using the wii balance board: a randomized, crossover pilot study in multiple sclerosis. Neurorehabil Neural Repair 27:516-525

52. Hoang P, Schoene D, Gandevia S et al (2016) Effects of a home-based step training programme on balance, stepping, cognition and functional performance in people with multiple sclerosis: a randomized controlled trial. Mult Scler J 22:94-103

53. Turner AP, Hartoonian N, Sloan AP et al (2016) Improving fatigue and depression in individuals with multiple sclerosis using telephone-administered physical activity counseling. J Consult Clin Psychol 84:297-309

54. Charvet LE, Yang J, Shaw MT et al (2017) Cognitive function in multiple sclerosis improves with telerehabilitation: results from a randomized controlled trial. PLoS ONE 12:e0177177

55. Stuifbergen AK, Becker H, Perez F et al (2012) A randomized controlled trial of a cognitive rehabilitation intervention for persons with multiple sclerosis. Clin Rehabil 26:882-893

56. Di Tella S, Pagliari C, Blasi V et al (2019) Integrated telerehabilitation approach in multiple sclerosis: a systematic review and meta-analysis. J Telemed Telecare 26:385-399

57. Molinaro A, Micheletti S, Pagani F et al (2020) Action observation treatment in a tele-rehabilitation setting: a pilot study in children with cerebral palsy. Disabil Rehabil. https://doi. org/10.1080/09638288.2020.1793009
58. Kim W-S, Lee K, Kim S et al (2019) Transcranial direct current stimulation for the treatment of motor impairment following traumatic brain injury. J Neuroeng Rehabil. https://doi.org/10.1186/ s12984-019-0489-9

59. Liberatore G, Clarelli F, Nuara A et al (2014) Predictors of effectiveness of multidisciplinary rehabilitation treatment on motor dysfunction in multiple sclerosis. Mult Scler J 20:862-870

60. Tremblay L, Esculier J, Vaudrin J et al (2012) Home-based balance training programme using Wii Fit with balance board for Parkinsons's disease: a pilot study. J Rehabil Med 44:144-150

61. Winstein CJ, Wolf SL, Dromerick AW et al (2016) Effect of a task-oriented rehabilitation program on upper extremity recovery following motor stroke: the ICARE randomized clinical trial. JAMA 315:571

62. Buccino G (2014) Action observation treatment: a novel tool in neurorehabilitation. Philos Trans R Soc Lond B Biol Sci 369:20130185

63. Ertelt D, Small S, Solodkin A et al (2007) Action observation has a positive impact on rehabilitation of motor deficits after stroke. NeuroImage 36(Suppl 2):T164-173

64. Pelosin E, Avanzino L, Bove M et al (2010) Action observation improves freezing of gait in patients with Parkinson's disease. Neurorehabil Neural Repair 24:746-752

65. Rocca MA, Meani A, Fumagalli S et al (2018) Functional and structural plasticity following action observation training in multiple sclerosis. Mult Scler J. https://doi.org/10.1177/1352458518 792771

66. Castiello U, Ansuini C, Bulgheroni M et al (2009) Visuomotor priming effects in Parkinson's disease patients depend on the match between the observed and the executed action. Neuropsychologia 47:835-842

67. Errante A, Di Cesare G, Pinardi C et al (2019) Mirror neuron system activation in children with unilateral cerebral palsy during observation of actions performed by a pathological model. Neurorehabil Neural Repair 33:419-431

68. Bergquist TF, Malec JF (1997) Psychology: current practice and training issues in treatment of cognitive dysfunction. NeuroRehabilitation 8:49-56

69. Mantovani E, Zucchella C, Bottiroli S et al (2020) Telemedicine and virtual reality for cognitive rehabilitation: a roadmap for the COVID-19 pandemic. Front Neurol. https://doi.org/10.3389/ fneur.2020.00926

70. van der Linden SD, Sitskoorn MM, Rutten G-JM et al (2018) Feasibility of the evidence-based cognitive telerehabilitation program remind for patients with primary brain tumors. J Neurooncol 137:523-532

71. Meltzer JA, Baird AJ, Steele RD et al (2018) Computer-based treatment of poststroke language disorders: a non-inferiority study of telerehabilitation compared to in-person service delivery. Aphasiology 32:290-311

72. Yeroushalmi S, Maloni H, Costello K et al (2020) Telemedicine and multiple sclerosis: a comprehensive literature review. J Telemed Telecare 26:400-413

73. McCallum S, Boletsis C (2013) Dementia games: a literature review of dementia-related serious games. In: Ma M, Oliveira MF, Petersen S (eds) Serious games development and applications. Springer, Berlin, pp 15-27

74. van der Kuil MNA, Visser-Meily JMA, Evers AWM et al (2018) A usability study of a serious game in cognitive rehabilitation: a compensatory navigation training in acquired brain injury patients. Front Psychol. https://doi.org/10.3389/fpsyg .2018 .00846

75. Lopes S, Magalhães P, Pereira A et al (2018) Games used with serious purposes: a systematic review of interventions in patients with cerebral palsy. Front Psychol. https://doi.org/10.3389/fpsyg .2018 .01712 
76. Bayley MT, Hurdowar A, Teasell R et al (2007) Priorities for stroke rehabilitation and research: results of a 2003 Canadian Stroke Network Consensus Conference. Arch Phys Med Rehabil 88:526-528

77. Wendebourg MJ, Heesen C, Finlayson M et al (2017) Patient education for people with multiple sclerosis-associated fatigue: a systematic review. PLoS ONE 12:e0173025

78. Jakimovski D, Weinstock-Guttman B, Gandhi S et al (2019) Dietary and lifestyle factors in multiple sclerosis progression: results from a 5-year longitudinal MRI study. J Neurol 266:866-875

79. Nag N, Jelinek GA (2019) A narrative review of lifestyle factors associated with parkinson's disease risk and progression. Neurodegener Dis 19:51-59

80. Levac DE, Huber ME, Sternad D (2019) Learning and transfer of complex motor skills in virtual reality: a perspective review. J NeuroEngineering Rehabil. https://doi.org/10.1186/s1298 4-019-0587-8

81. Menin A, Torchelsen R, Nedel L (2018) An analysis of VR technology used in immersive simulations with a serious game perspective. IEEE Comput Graph Appl 38:57-73

82. Maggio MG, Maresca G, De Luca R et al (2019) The growing use of virtual reality in cognitive rehabilitation: fact, fake or vision? a scoping review. J Natl Med Assoc 111:457-463

83. Li Z, Han XG, Sheng J et al (2016) Virtual reality for improving balance in patients after stroke: a systematic review and metaanalysis. Clin Rehabil 30:432-440

84. Piron L, Turolla A, Agostini M et al (2009) Exercises for paretic upper limb after stroke: A combined virtual-reality and telemedicine approach. J Rehabil Med 41:1016-1020

85. Lohse KR, Hilderman CGE, Cheung KL et al (2014) Virtual reality therapy for adults post-stroke: a systematic review and meta-analysis exploring virtual environments and commercial games in therapy. PLoS ONE. https://doi.org/10.1371/journ al.pone.0093318

86. Merians AS, Poizner H, Boian R et al (2006) Sensorimotor training in a virtual reality environment: does it improve functional recovery poststroke? Neurorehabil Neural Repair 20:252-267

87. Cikajlo I, Peterlin PK (2019) Advantages of using 3D virtual reality based training in persons with Parkinson's disease: a parallel study. J Neuroeng Rehabil 16:1-14

88. Maggio MG, Russo M, Cuzzola MF et al (2019) Virtual reality in multiple sclerosis rehabilitation: a review on cognitive and motor outcomes. J Clin Neurosci. https://doi.org/10.1016/j. jocn.2019.03.017

89. Golomb MR, Warden SJ, Fess E et al (2011) Maintained hand function and forearm bone health 14 months after an in-home virtual-reality videogame hand telerehabilitation intervention in an adolescent with hemiplegic cerebral palsy. J Child Neurol 26:389-393

90. Golomb MR, McDonald BC, Warden SJ et al (2010) In-home virtual reality videogame telerehabilitation in adolescents with hemiplegic cerebral palsy. Arch Phys Med Rehabil 91(1-8):e1

91. Howard MC (2017) A meta-analysis and systematic literature review of virtual reality rehabilitation programs. Comput Hum Behav 70:317-327
92. Chang E, Kim HT, Yoo B (2020) Virtual reality sickness: a review of causes and measurements. Int J Hum-Comput Interact 36:1658-1682

93. Bedwell WL, Pavlas D, Heyne K et al (2012) Toward a taxonomy linking game attributes to learning: an empirical study. Simul Gaming 43:729-760

94. Gotz U, Brutsch K, Bauer R, et al (2011) A virtual reality system for robot-assisted gait training based on game design principles. In: 2011 International Conference on Virtual Rehabilitation. Zurich, Switzerland: IEEE pp 1-2.

95. Heiden E, Lajoie Y (2010) Games-based biofeedback training and the attentional demands of balance in older adults. Aging Clin Exp Res 22:367-373

96. Yen C-Y, Lin K-H, Hu M-H et al (2011) Effects of virtual reality-augmented balance training on sensory organization and attentional demand for postural control in people with parkinson disease: a randomized controlled trial. Phys Ther 91:862-874

97. Cappa P, Clerico A, Nov O et al (2013) Can force feedback and science learning enhance the effectiveness of neuro-rehabilitation? An experimental study on using a low-cost $3 \mathrm{D}$ joystick and a virtual visit to a zoo. PLoS ONE 8:e83945-e83945

98. Nijenhuis SM, Prange GB, Amirabdollahian F et al (2015) Feasibility study into self-administered training at home using an arm and hand device with motivational gaming environment in chronic stroke. J NeuroEngineering Rehabil. https://doi. org/10.1186/s12984-015-0080-y

99. Standen PJ, Brown DJ, Battersby S et al (2011) A study to evaluate a low cost virtual reality system for home based rehabilitation of the upper limb following stroke. Int J Disabil Hum Dev $10: 337-341$

100. Wittmann F, Held JP, Lambercy O et al (2016) Self-directed arm therapy at home after stroke with a sensor-based virtual reality training system. J NeuroEngineering Rehabil. https://doi. org/10.1186/s12984-016-0182-1

101. Da Gama A, Fallavollita P, Teichrieb V et al (2015) Motor rehabilitation using kinect: a systematic review. Games Health J 4:123-135

102. Galen SS, Clarke CJ, Allan DB et al (2011) A portable gait assessment tool to record temporal gait parameters in SCI. Med Eng Phys 33:626-632

103. Jagos H, David V, Haller M et al (2015) A framework for (tele-) monitoring of the rehabilitation progress in stroke patients. Appl Clin Inform 6:757-768

104. Kuznetsov NA, Robins RK, Long B et al (2018) Validity and reliability of smartphone orientation measurement to quantify dynamic balance function. Physiol Meas 39:02NT01

105. Hou YR, Chiu YL, Chiang SL et al (2020) Development of a smartphone-based balance assessment system for subjects with stroke. Sens Switz. https://doi.org/10.3390/s20010088

106. Insel TR (2017) Digital phenotyping: technology for a new science of behavior. JAMA 318:1215

107. Holmqvist LW (2001) Environmental factors in stroke rehabilitation. BMJ 322:1501-1502 